\title{
Biomechanical Engineering Analysis of Glaucoma Micro Stent Implant with Check Valve for Intraocular Pressure Reduction (IOP) by Finite Element Method (FEM)
}

\author{
Ö Karaçalı* \\ Department of Mechanical Engineering, Istanbul University, Turkey
}

Received: 㸷 March 13, 2018; Published: 制 March 20, 2018

*Corresponding author: Özdoğan Karaçall, Department of Mechanical Engineering, Faculty of Engineering, Istanbul University, Avcılar, Istanbul, Turkey, Tel: 9050521055 30; Email: ozdogank@istanbul.edu.tr

\begin{abstract}
Biomechanical factors affect the design model of glaucoma micro stent (GMS) geometry, material and shape in correcting treatment of high intraocular pressure (IOP). The goal of this research was to characterize the biomechanics of the GMS using computational finite element method. In this research, the model for nano-composite materials will be investigated for analyzing the material behavior of the GMS with check valve used in the intraocular fluid evacuation system which is not made until now to control the intraocular pressure. It has not been reported about what impacts on GMS with check valve design and material selection to ensure discharge of ocular fluid in intraocular pressure (IOP), ensuring fluid flow continuity. Finite element Analysis simulation methodology for realization of accurate structural and aqueous humor (AH) behavior micro model during this analysis provided valuable results of useful data of GMS material behavior that are time consuming and expensive to determine otherwise. The check valve provided a control mechanism for AH pressure to regulate the IOP. For this reason, material deformation von-Mises and fluid flow behaviors model was developed. To achieve these, finite element analysis were employed to calculate compressive stresses and equivalent von-Mises stress criteria as well as the analysis by the strength of material's experimental procedure in laboratory conditions. A finite element analysis (FEA) of GMS under very low cyclic loading conditions is presented. The results of modeling were verified with experimental data for biocompatible and biodegradable GMS from nano-composite material. This research provided a data set for preventing glaucoma disease and improving intraocular tension by new design of GMS.
\end{abstract}

Keywords: Glaucoma micro stent; Finite Element Analysis (FEA); Biomechanics; Von-Mises stress; Strain

Abbreviations: GMS: Glaucoma Micro Stent; IOP: Intraocular Pressure; AH: Aqueous Humor; FEA: Finite Element Analysis; FE: Finite Element

\section{Introduction}

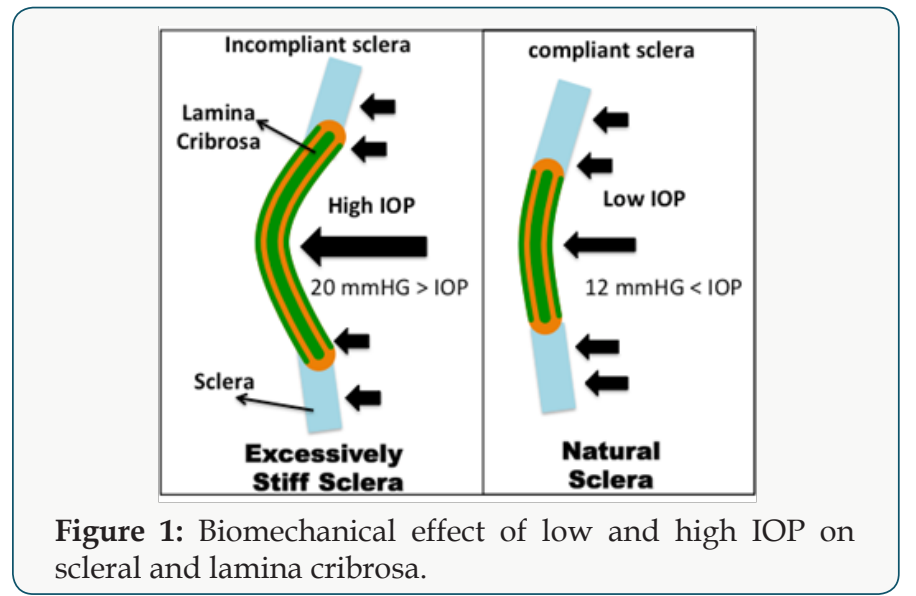

The drainage micro stent is a new glaucoma ocular tension treatment and has been developed primarily to correct the deteriorated intraocular fluid circulation in glaucoma disease [1]. Glaucoma is an ocular disease resulting in progressive and irreversible loss of vision by damaged optic nerve or complete blindness [2]. Currently, the only clinically proven treatment for slowing or halting the diseases progress is lowering IOP by surgical or pharmaceutical means $[3,4]$. Biomechanical models can help understand how the mechanical changes are transformed into a biological response of the tissue [5]. LC biomechanics can depend on the compliance of the sclera and on IOP as shown in (Figure 1). An increase of IOP in compliant sclera leads to large scleral deformations and a large expansion of the scleral canal, pulling the LC taut. A rigid sclera 
distorts only slightly under increased IOP, with related minor scleral canal growth and slight extending of the LC [6].

The purpose of the research explained in the paper is to evaluate the changes in aqueous humor dynamics and the efficacy and safety of the new glaucoma micro stent (GMS) with check valve developed. The modified new designed implant GMS shown in (Figure 2) helps thousands of people successfully cope with complications such as tube hypotony due to IOP [7]. Polymeric microfluidic check valve is a device that provides opening a forward fluid flow or closing for a reverse flow in GMS and was used for biomedical analysis. In this research, polymer one way check-valve for controlling microfluidic flow was used to control the AH The GMS is becoming increasingly popular for patients undergoing glaucoma surgery. Some consider this implant as an integral component of modified "trabeculectomy." All trabecular tissue remains in place and aqueous outflow is maintained through the lumen of the implant [8]. Although the implantation of GMS is fairly straightforward, there are some key technical aspects for correct implant position in the anterior chamber, including adequate tube clearance from the cornea and iris [9]. This research examines the feasibility of using factors to examine the position and function of the GMS. Figure 2 shows example glaucoma micro stent developed for experimental study.

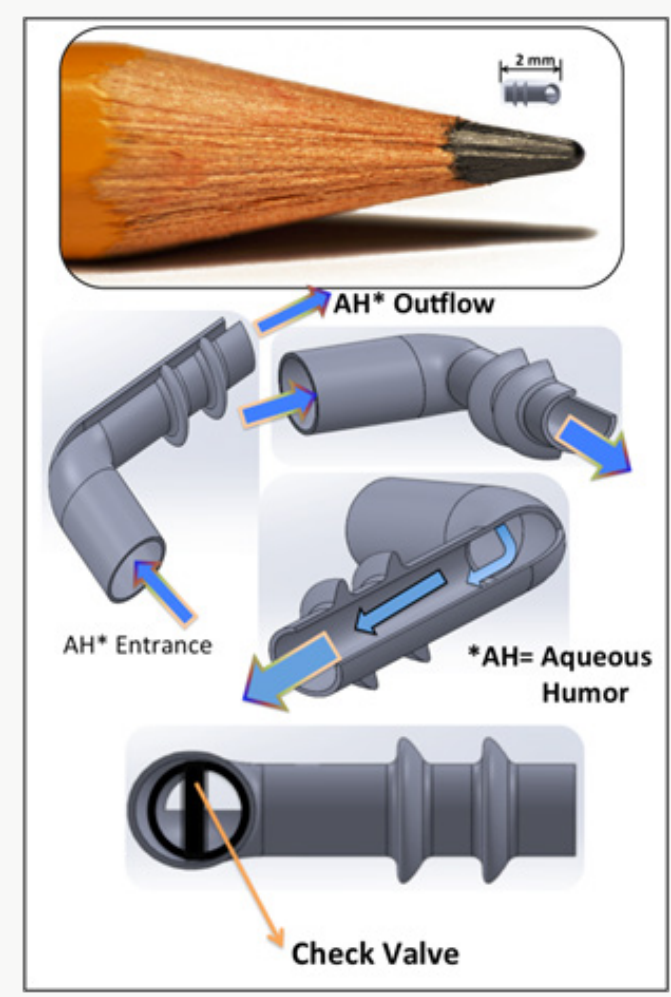

Figure 2: Glaucoma micro stent model.

\section{Materials and Methods: Biomechanical Models of GMS}

The finite element analysis were employed to calculate compressive stresses and equivalent Von Mises stresses as well as the analysis by the strength of materials experimental procedure
[9]. In this study, the Ansys software simulation modelling of the drainage tube GMS was used as shown in (Figure. 3). A flow model for pressure and velocity values and geometric shape and material properties in (Figure.3) was examined by finite element analysis [9] and computational fluid dynamics. FE models of the GMS was generated and resolved by the commercially available Ansys program. Proper fluid passageway geometry within the GMS, an axisymmetric 2D mesh was employed. The mesh included 10,518 two-dimensional elements. Displacement stresses of glaucoma micro stent are displayed in (Figure 4). For the GMS implant tube area, a 3D mesh was formed including 150,937 hexahedral elements. Aqueous humor was accepted as an incompressible Newtonian fluid. Aqueous Humor flows in the GMS with rate of 8 $\mu \mathrm{L} /$ minute and was aimed to develop a new check valve which will facilitate elimination of the discharge of the eye liquid through the glaucoma micro stent in Figure 2. This check valve will control a normal mature eye pressure of $11 \mathrm{mmHg}$ and $18 \mathrm{mmHg}$ pressure. If the blood pressure drops below $8 \mathrm{mmHg}$, the stent will close and the eye will check for low aquoes humor pressure. When $21 \mathrm{mmHg}$ and above ocular pressure is established in the same way, the valve in the stent canal will be opened and the eye liquid will be ejected. A study of this kind of approach is yet to be found in the international literature. This application was done by creating a fully artificial chamber of flow.
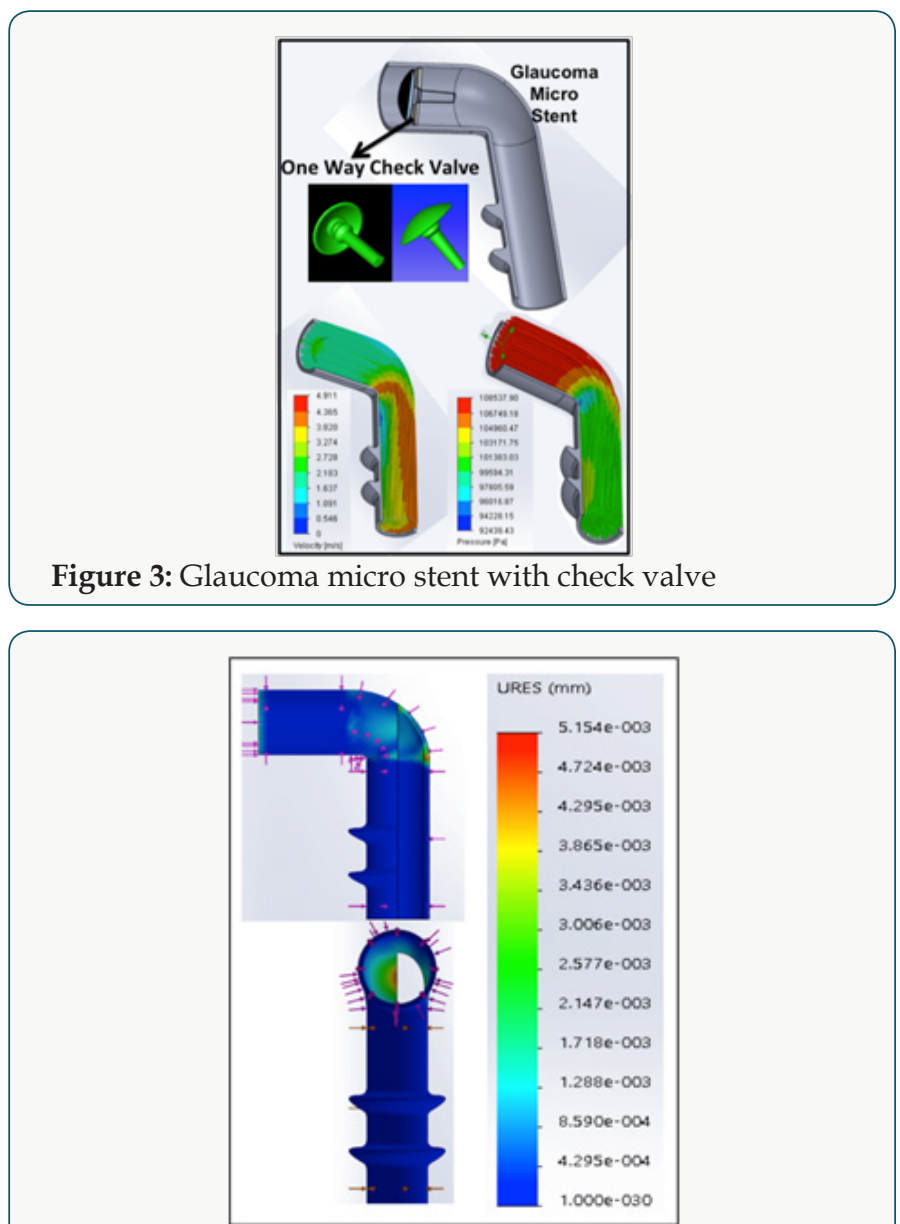

Figure 4: Displacement stress of glaucoma micro stent. 
The small tube with overall outflow resistances and computational fluid dynamics were calculated and verified by the engineering analyses (Hagen-Poiseuille) [10]. The size of lumen in GMS is limited to permit adequate resistance based on HagenPoiseuille calculation to avoid hypotony shown in (Figure. 5).

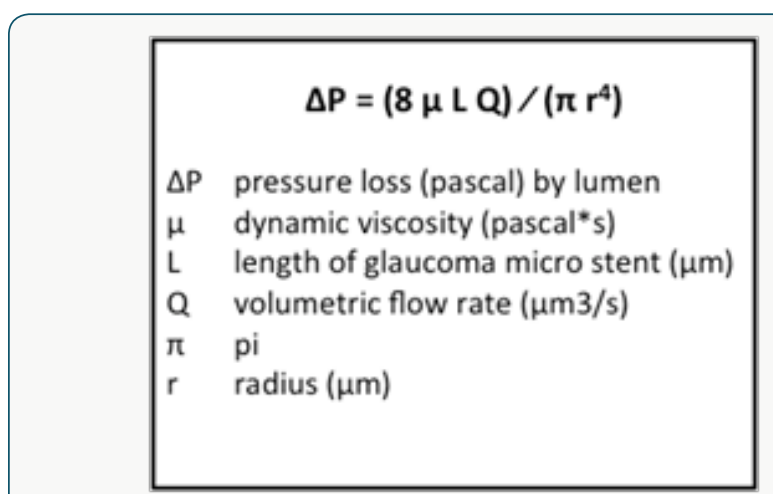

Figure 5: Hagen-Poiseuille AH flow resistance

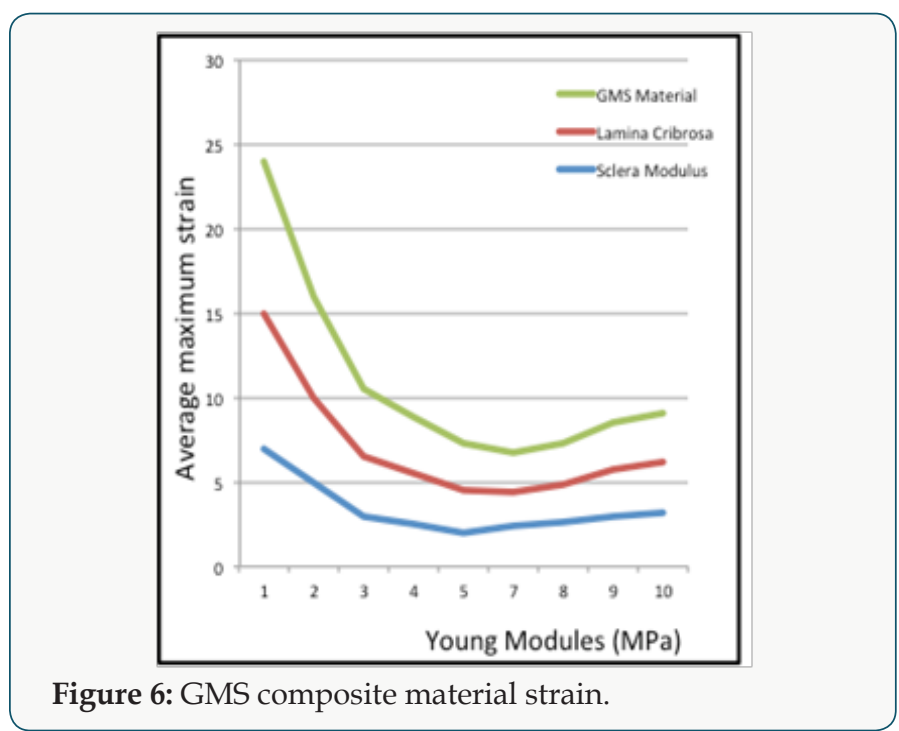

The IOP amount depends on the tube fluidic resistance. The fluidic resistance changed due to cross-sectional area and compression level of the tube. The mechanical response to IOP depends on the individual geometry as well as the mechanical properties of the eye. Stress-force per unit area- and strain- relative linearly proportional deformation is associated to each other through material properties [9]. In living tissue, however, several complexities, such as nonlinearity and anisotropy, have to be considered. Corresponding to the stress, the maximum strain was also greatest but strain distribution differed slightly from stress because of variations in tissue Young's modulus as displayed in (Figure 6). The finite element (FE) method enabled for calculating the deformation of an object if the geometry, material properties, the mechanical loading of the GMS. These biomechanical models are categorized as general or eye-specific models. The biomechanical reaction of GMS results encouraged to construct the FE model including the effect of the scleral channel size. The models from Sigal et al. also showed that the ocular surface tissues are introduced to several kinds of stress and strain, shearing, compressive and tensile. In all eye samples of the compressive strains levels are higher than those have shearing and tensile strains $[10,11]$.

The high stress zones were detected in the junction of the GMS. In the tube entrance zone, the boundary stress declines from inlet to outflow, and rises at the check valve area (Figure 7). indicates that the maximum stress to include the von Mises stress combined configuration with sclera tissue in the research model was $150 \mathrm{MPa}$ (ultra tensile strength). A pressure von Mises criterion calculating a nonlinear dependence of the yield stress on the hydrostatic stress was tested to GMS composite polymer as given in (Figure 8). The stress conditions affected to a mode of uniaxial tensile stress are related with a tri-axial tensile stress state.

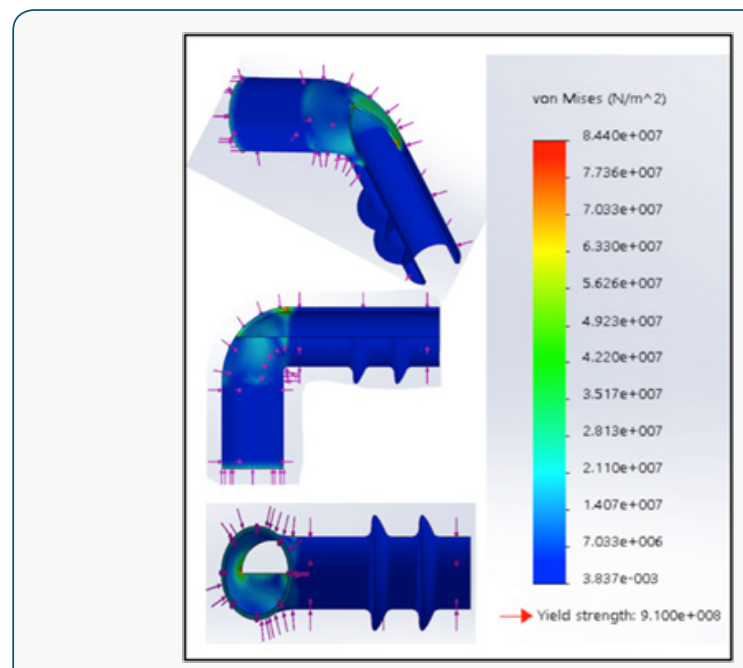

Figure 7: Von Mises Stresses on the glokom micro stent.

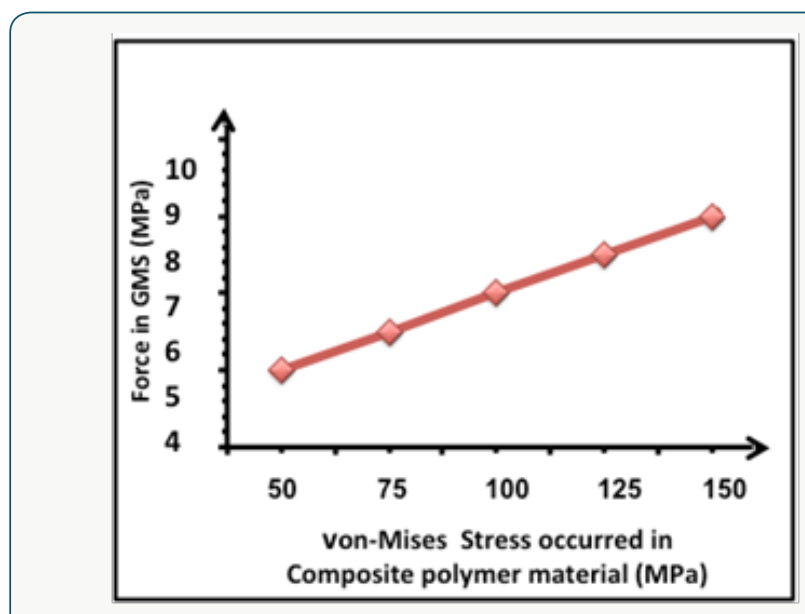

Figure 8: von-Mises stress in GMS under different forces.

\section{Conclusion}

This study investigates geometry and material under stresses and its effects on GMS for significant reduction of IOP without medication burden in open angle glaucoma. The computational modelling of FEM with accurate geometry and material properties was used for a biocompatible GMS. This innovation will create a unique work by providing an environment for glaucoma micro 
stent to clog and to automatically clean the channel itself. GMS was designed to improve aqueous outflow and reduce IOP conclusion to date indicate that the GMS implant is feasible, safe and capable of significant reduction in IOP.

\section{Acknowledgement}

This research is funded by Istanbul University, Turkey, (The Scientific Research Foundation). Such support is greatly acknowledged.

\section{References}

1. Christakis PG, Tsai JC, Kalenak JW (2013) The Ahmed versus Baerveldt study: three-year treatment outcomes. Ophthalmology 120(11): 22322240 .

2. Barton K, Feuer WJ, Budenz DL, Schiffman J, Costa VP, et al. (2014) Three-year treatment outcomes in the Ahmed Baerveldt comparison study. Ophthalmology 121(8): 1547-1557.

3. Demetrios T Manasses, Leon Au (2016) The New Era of Glaucoma Microstent Surgery Ophthalmology 5: 135-146.

4. Sherwood JM, Reina Torres E, Bertrand JA, Rowe B (2016) Measurement of outflow facility using iPerfusion. PLoS One 11: 599-694.
5. Karaçali Ö (2014) Structured Analysis of Stainless Steel 316L Material of Micro-Forceps Used in Vitrectomy Surgery by Biomechanics Modeling. Acta physica polonica A 125(2): 180-182.

6. Reina Torres E, Wen JC, Liu KC (2017) VEGF as a paracrine regulator of conventional outflow facility. Invest Ophthalmol Vis Sci 58: 1899-1908.

7. Millar JC, Clark AF, Pang IH (2011) Assessment of aqueous humor dynamics in the mouse by a novel method of constant-flow infusion. Invest Ophthalmol Vis Sci 52(2): 685-694.

8. Bahler CK, Hann CR, Fjield T, Haffner D, Heitzmann H, et al. (2012) Second-generation trabecular meshwork bypass stent (iStent inject) increases outflow facility in cultured human anterior segments. Am J Ophthalmol 153(6): 1206-1213.

9. Karaçali Ö (2015) Fatigue Analysis of Ti-55 Alloy Material for Multifunctional Surgical Instruments in Medical Engineering. Acta physica polonica A 127(4):1199-1201.

10. Mortensen NA, Okkels F, Bruus H (2005) Reexamination of HagenPoiseuille flow: shape dependence of the hydraulic resistance in microchannels. Phys Rev E Stat Nonlin Soft Matter Phys 71: 2-5.

11. Sigal IA, Ethier CR (2009) Biomechanics of the optic nerve head. Experimental Eye Research 88(4): 799-807.
To Submit Your Article Click Here:

This work is licensed under Creative Commons Attribution 4.0 License

Submit Article

DOI: $10.32474 /$ OAJBEB.2018.01.000122

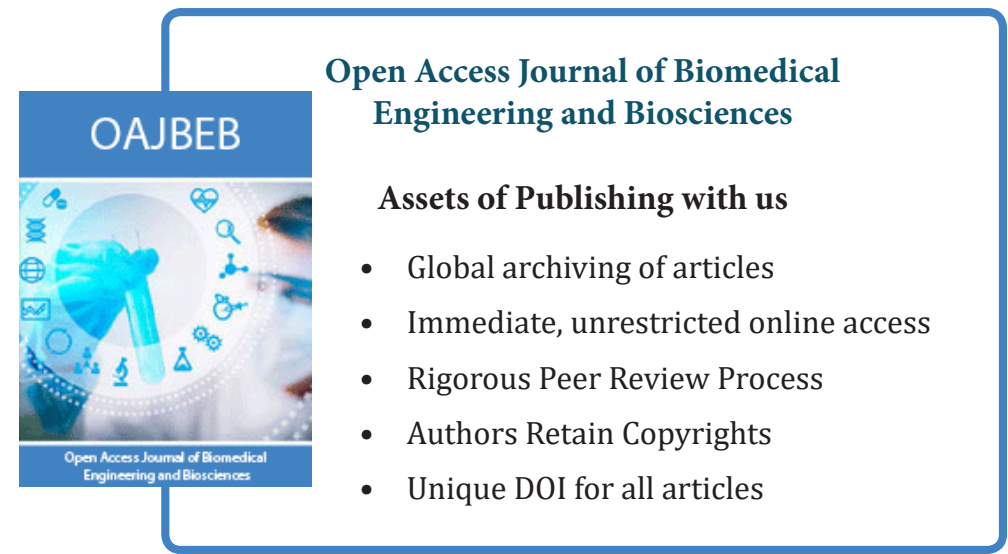

